\title{
Sigma-additivity of quasi-measure extensions of a
}

\section{measure}

\author{
D. Bogner and R. Denk
}

\begin{abstract}
The situation in which every quasi-measure extension of a given measure is $\sigma$-additive is characterized in the case of an atomic or two-valued measure.
\end{abstract}

Subject Classifications: 28A12

\section{Introduction}

In general the existence of measure extensions or extremal measure extensions is not guaranteed and can be proved only in special cases (cf. [2], [3]). Quite different is the situation if we look for quasi-measure extensions of a given measure. It is well-known that extensions and even extreme extensions always exist (see [1], section 3.4, [13], p. 194); the set of all quasi-measure extensions is compact and an integral representation holds, see below. This paper deals with the question in which cases every quasi-measure extension of a given measure is $\sigma$-additive. In section 2 we give a characterization of that situation in the case where the smaller $\sigma$-algebra is atomic with respect to the measure. A special case of this is a $\sigma$-algebra which is generated by a countable partition. In section 3 these results are applied to twovalued measures. Most results in sections 2 and 3 are part of the diploma thesis of the first author [4] which was finished under guidance of Prof. D. Bierlein at the University of Regensburg.

We fix the following notations (cf. [1],[11]): Let $\mathcal{A}$ and $\mathcal{A}_{1}$ be algebras [ $\sigma$-algebras] of subsets of a nonempty set $\Omega$ with $\mathcal{A} \subset \mathcal{A}_{1}$. For $\mathcal{E} \subset 2^{\Omega}$ we denote by $\mathcal{E}_{\beta}$ the $\sigma$-algebra generated by $\mathcal{E}$. $b a(\mathcal{A})[c a(\mathcal{A})]$ is defined as the Banach lattice of all real-valued bounded additive $[\sigma$-additive $]$ set functions on $\mathcal{A}$, and we call the elements of the positive cone $b a_{+}(\mathcal{A})$ quasi-measures and the elements of $c a_{+}(\mathcal{A})$ measures.

For $\nu \in b a_{+}(\mathcal{A})$ we set $R(\nu):=\{\nu(A): A \in \mathcal{A}\}$ and

$$
E\left(\nu, \mathcal{A}_{1}\right):=\left\{\mu \in b a_{+}\left(\mathcal{A}_{1}\right): \mu \mid \mathcal{A}=\nu\right\}
$$

For a measure $\nu \in c a_{+}(\mathcal{A})$ we define $E_{\sigma}\left(\nu, \mathcal{A}_{1}\right):=E\left(\nu, \mathcal{A}_{1}\right) \cap c a\left(\mathcal{A}_{1}\right) . \quad b a(\mathcal{A})$ is isometrically isomorphic to the topological dual space of $B(\mathcal{A})$ where $B(\mathcal{A})$ is defined 
as the closure of the $\mathcal{A}$-measurable simple functions in the Banach space of all bounded real-valued functions on $\Omega$ with the supremum-norm (see [5], section IV.5). This allows us to define the weak*-topology on $b a(\mathcal{A})$. In the following $b a(\mathcal{A})$ will always be endowed with this topology.

In many cases the $\sigma$-additivity of all elements of some subset of $b a(\mathcal{A})$ can be reduced to the $\sigma$-additivity of the extremal points of this subset. This general principle follows from the Choquet theory and is stated in the following lemma where ex $M$ stands for the set of all extremal points of $M$ :

Lemma 1 Let $\mathcal{A}$ be an algebra and $M$ a compact convex subset of ba $(\mathcal{A})$. Then we have $M \subset c a(\mathcal{A})$ if and only if $\operatorname{ex} \subset \subset c a(\mathcal{A})$.

Proof. Only the if-part has to be proved. Let $\mu \in M$ and let $\mathcal{B}_{0}(M)$ be the Baire$\sigma$-algebra of $M$. Then the theorem of Choquet-Bishop-de Leeuw (see [12], p. 30) tells us that there exists a measure $m: \mathcal{B}_{0}(M) \cap \operatorname{ex} M \rightarrow[0,1]$ with $m(\operatorname{ex} M)=1$, for which we have $f(\mu)=\int_{\mathrm{ex} M} f(\nu) d m(\nu)$ for every continous linear functional $f$ on $b a(\mathcal{A})$. In particular, we get for every $A \in \mathcal{A}$

$$
\mu(A)=\int_{\operatorname{ex} M} \nu(A) d m(\nu)
$$

For a sequence $A_{1} \subset A_{2} \subset \ldots$ with $A:=\bigcup_{n \in \mathbb{N}} A_{n} \in \mathcal{A}$ we obtain from ex $M \subset$ $c a(\mathcal{A})$ that $\nu\left(A_{n}\right) \rightarrow \nu(A)$ for all $\nu \in$ ex $M$. Because of the compactness of $M$ there exists a constant $c>0$ with $\left|\nu\left(A_{n}\right)\right| \leq|\nu|(\Omega) \leq c$ for all $n \in \mathbb{N}$ and $\nu \in$ ex $M$. Using Lebesgue's convergence theorem, we see $\mu\left(A_{n}\right)=\int_{\operatorname{ex~} M} \nu\left(A_{n}\right) d m(\nu) \rightarrow$ $\int_{\mathrm{ex} M} \nu(A) d m(\nu)=\mu(A)$ and therefore $\mu \in c a(\mathcal{A})$.

Examples for the set $M$ in Lemma 1 are $E\left(\nu, \mathcal{A}_{1}\right)$ for some given $\nu \in b a_{+}(\mathcal{A})$, see the next sections, and the core $C(v)$ of a game $(\Omega, \mathcal{A}, v)$ as defined in [6].

\section{Extensions of atomic measures}

For an algebra $\mathcal{A}$ and a system $\mathcal{E} \subset \mathcal{A}$ we call an element $A \in \mathcal{A} \backslash \mathcal{E}$ an $\mathcal{E}$-atom if for every $B \in \mathcal{A}$ with $B \subset A$ we have $B \in \mathcal{E}$ or $A \backslash B \in \mathcal{E}$ (cf. [9]). $\mathcal{A}$ is called $\mathcal{E}$-atomic if for every $A \in \mathcal{A} \backslash \mathcal{E}$ there exists an $\mathcal{E}$-atom $B \subset A$. For $\mathcal{E}=\{\emptyset\}$ we obtain the classical definitions of an atom and an atomic algebra. For $\nu \in b a_{+}(\mathcal{A})$ we define $\mathcal{N}(\nu):=\left\{A \subset \Omega: \nu^{*}(A)=0\right\}$ (cf. [1], section 4.2). An algebra $\mathcal{A}$ is called $\nu$-atomic if it is $(\mathcal{N}(\nu) \cap \mathcal{A})$-atomic. We set $a t(\mathcal{A}):=\left\{\nu \in b a_{+}(\mathcal{A}): \mathcal{A}\right.$ is $\nu$-atomic $\}$ and call the elements of $a t(\mathcal{A})$ atomic quasi-measures. In the following lemma, countable means finite or countably infinite.

Lemma 2 Let $\mathcal{A}$ be a $\sigma$-algebra and $\nu \in$ at $(\mathcal{A})$. Then there exists a countable family of disjoint $\nu$-atoms whose union is $\Omega$. 
Proof. By $A \sim B: \Leftrightarrow \nu(A \triangle B)=0$ an equivalence relation is defined on the $\nu$-atoms of $\mathcal{A}$. Let $\mathcal{K}^{\prime}$ be a system of representatives of this equivalence relation. It is easily seen that for every $n \in \mathbb{N}$ there is only a finite number of $K^{\prime} \in \mathcal{K}^{\prime}$ with $\nu\left(K^{\prime}\right)>\frac{1}{n}$. Thus $\mathcal{K}^{\prime}$ is countable. Let $N \subset \mathbb{N}$ with $\mathcal{K}^{\prime}=\left\{K_{n}^{\prime}: n \in N\right\}$ and $n_{0} \in N$. Then by $K_{n}:=$ $K_{n}^{\prime} \backslash \bigcup\left\{K_{j}^{\prime}: j<n, j \in N\right\}$ and $\mathcal{K}:=\left\{K_{n}: n \in N \backslash\left\{n_{0}\right\}\right\} \cup\left\{K_{n_{0}} \cup\left(\Omega \backslash \bigcup_{n \in N} K_{n}\right)\right\}$ a partition of $\Omega$ with the desired properties is defined.

Now we consider two $\sigma$-algebras $\mathcal{A} \subset \mathcal{A}_{1}$ and some $\nu \in c a_{+}(\mathcal{A})$. If $\nu$ is an atomic measure, the following theorem gives some conditions under which each quasi-measure extension of $\nu$ is $\sigma$-additive. Condition (iii) and some simplifications in the proof of this theorem are due to Z. Lipecki [8].

Theorem 1 Let $\mathcal{A} \subset \mathcal{A}_{1}$ be $\sigma$-algebras and $\nu \in c a_{+}(\mathcal{A}) \cap$ at $(\mathcal{A})$. Then the following conditions are equivalent:

(i) $E\left(\nu, \mathcal{A}_{1}\right)=E_{\sigma}\left(\nu, \mathcal{A}_{1}\right)$.

(ii) $\operatorname{ex} E\left(\nu, \mathcal{A}_{1}\right) \subset E_{\sigma}\left(\nu, \mathcal{A}_{1}\right)$.

(iii) For each increasing sequence $A_{1} \subset A_{2} \subset \ldots$ in $\mathcal{A}_{1}$ with $\Omega=\bigcup_{n \in \mathbb{N}} A_{n}$ we have $\nu_{*}\left(A_{n}\right) \rightarrow \nu(\Omega)(n \rightarrow \infty)$.

(iv) $E\left(\nu, \mathcal{A}_{1}\right) \cap a t\left(\mathcal{A}_{1}\right) \subset E_{\sigma}\left(\nu, \mathcal{A}_{1}\right)$.

(v) $E\left(\nu, \mathcal{A}_{1}\right) \cap\left\{\mu \in \operatorname{at}\left(\mathcal{A}_{1}\right): R(\mu)=R(\nu)\right\} \subset E_{\sigma}\left(\nu, \mathcal{A}_{1}\right)$.

(vi) For each $\nu$-atom $A$ and each disjoint sequence $\left(A_{n}\right)_{n \in \mathbb{N}}$ in $\mathcal{A}_{1}$ with $A=\bigcup_{n \in \mathbb{N}} A_{n}$ there exists a finite $N \subset \mathbb{N}$ with $\nu_{*}\left(\bigcup_{n \in N} A_{n}\right)=\nu(A)$.

If one (and therefore each) of the above conditions is fulfilled, every $\mu \in E\left(\nu, \mathcal{A}_{1}\right)$ is atomic.

Proof. For general $\nu \in c a_{+}(\mathcal{A})$ the equivalence of $(i)$ and $(i i)$ follows from Lemma 1 and the equivalence of $(i i)$ and $(i i i)$ is shown in [6], Example 9. Now let $\nu \in$ $c a_{+}(\mathcal{A}) \cap a t(\mathcal{A})$. We show that $(v)$ implies $(i i)$ by proving

$$
\operatorname{ex} E\left(\nu, \mathcal{A}_{1}\right) \subset\left\{\mu \in \operatorname{at}\left(\mathcal{A}_{1}\right): R(\mu)=R(\nu)\right\}
$$

Indeed, let $\mu \in \operatorname{ex} E\left(\nu, \mathcal{A}_{1}\right)$ and $A \in \mathcal{A}_{1} \backslash \mathcal{N}(\mu)$. Due to a well-known theorem of Plachky ([13], Theorem 1) there exists a sequence $\left(A_{n}\right)_{n \in \mathbb{N}} \subset \mathcal{A}$ with $\mu\left(A \triangle A_{n}\right)<\frac{1}{n}$. Using the completeness of $\mathcal{A}$ with respect to the metric induced by $\mu \mid \mathcal{A}$, we obtain the existence of $\tilde{A} \in \mathcal{A}$ with $\mu(A \triangle \tilde{A})=0$. In particular, we have $\mu(A)=\nu(\tilde{A})$ and thus $R(\mu)=R(\nu)$. Because of $\nu \in \operatorname{at}(\mathcal{A})$ there exists a $\nu$-atom $K \subset \tilde{A}$ and we see from [14], p. 244, that $K \cap A$ is a $\mu$-atom. $(i) \Rightarrow(i v)$ and $(i v) \Rightarrow(v)$ are trivial and thus conditions $(i)$ to $(v)$ are equivalent.

$($ iii $) \Rightarrow(v i)$ : Let $A$ be a $\nu$-atom and $A=\bigcup_{n \in \mathbb{N}} A_{n}$ with disjoint $A_{n} \in \mathcal{A}_{1}$. For $A_{n}^{\prime}:=$ $\bigcup_{j \leq n} A_{j} \cup(\Omega \backslash A)$ condition (iii) implies $\nu_{*}\left(A_{n}^{\prime}\right) \rightarrow \nu(\Omega)$ and therefore $\nu_{*}\left(\bigcup_{j \leq n} A_{j}\right) \rightarrow$ $\nu(\bar{A})$. Because $A$ is a $\nu$-atom there exists some $n \in \mathbb{N}$ with $\nu_{*}\left(\bigcup_{j \leq n} A_{j}\right)=\nu(A)$. 
(vi) $\Rightarrow($ iii $)$ : Let $A_{1} \subset A_{2} \subset \ldots$ be an increasing sequence in $\mathcal{A}_{1}$ with $\Omega=\bigcup_{n \in \mathbb{N}} A_{n}$ and $\mathcal{K} \subset \mathcal{A}$ a countable partition of $\Omega$ consisting of $\nu$-atoms, see Lemma 2. According to $(v i)$ for every $\nu$-atom $K \in \mathcal{K}$ there exists an $n \in \mathbb{N}$ with $\nu(K)=\nu_{*}\left(K \cap A_{n}\right)$. Therefore for every finite $\mathcal{K}^{\prime} \subset \mathcal{K}$ we have some $n \in \mathbb{N}$ with $\nu_{*}\left(A_{n}\right) \geq \nu_{*}\left(\bigcup_{K \in \mathcal{K}^{\prime}}(K \cap\right.$ $\left.\left.A_{n}\right)\right)=\nu\left(\bigcup_{K \in \mathcal{K}^{\prime}} K\right)$. Using the $\sigma$-additivity of $\nu$, we conclude that $\nu_{*}\left(A_{n}\right) \rightarrow \nu(\Omega)$. Now let one (and therefore each) of the conditions $(i)$ to $(v i)$ be fulfilled and $\mu \in$ $E\left(\nu, \mathcal{A}_{1}\right)=E_{\sigma}\left(\nu, \mathcal{A}_{1}\right)$. For $A \in \mathcal{A}_{1} \backslash \mathcal{N}(\mu)$ there exists a $K \in \mathcal{K}$ (where $\mathcal{K}$ is defined as above) with $\mu(A \cap K)>0$. Condition (vi) tells us that $\mu \mid \mathcal{A}_{1} \cap K \in$ at $\left(\mathcal{A}_{1} \cap K\right)$. Therefore there exists a $\mu$-atom $\tilde{A} \subset A \cap K$ and we obtain $\mu \in \operatorname{at}\left(\mathcal{A}_{1}\right)$.

Remark 1 The following example shows that the inclusions in

$$
\operatorname{ex} E\left(\nu, \mathcal{A}_{1}\right) \subset E\left(\nu, \mathcal{A}_{1}\right) \cap\left\{\mu \in \operatorname{at}\left(\mathcal{A}_{1}\right): R(\mu)=R(\nu)\right\} \subset E\left(\nu, \mathcal{A}_{1}\right) \cap a t\left(\mathcal{A}_{1}\right)
$$

(cf. (2) and condition $(v))$ can be proper: Let $\mathcal{A}=\{\emptyset,\{1,2\},\{3\},\{1,2,3\}\}, \nu(\{1,2\})=$ $\frac{2}{3}, \nu(\{3\})=\frac{1}{3}$ and $\mathcal{A}_{1}=2^{\{1,2,3\}}$. Then for $\mu_{1}$ defined by $\mu_{1}(\{n\}):=\frac{1}{3}(n \in\{1,2,3\})$ we have $\mu_{1} \in \operatorname{at}\left(\mathcal{A}_{1}\right) \cap\left(E\left(\nu, \mathcal{A}_{1}\right) \backslash \operatorname{ex} E\left(\nu, \mathcal{A}_{1}\right)\right)$ and $R\left(\mu_{1}\right)=R(\nu)$. Similarly $\mu_{2}$ with $\mu_{2}(\{1\})=\frac{1}{2}$ and $\mu_{2}(\{2\})=\frac{1}{6}$ is in $E\left(\nu, \mathcal{A}_{1}\right) \cap$ at $\left(\mathcal{A}_{1}\right)$ but $R\left(\mu_{2}\right) \neq R(\nu)$.

Corollary. Let $\mathcal{A} \subset \mathcal{A}_{1}$ be $\sigma$-algebras where $\mathcal{A}$ is generated by a countable partition of $\Omega$ and $\nu \in c a_{+}(\mathcal{A})$. Then $E\left(\nu, \mathcal{A}_{1}\right)=E_{\sigma}\left(\nu, \mathcal{A}_{1}\right)$ holds if and only if for every atom $A$ of $\mathcal{A}$ with $\nu(A)>0$ there exists an $n \in \mathbb{N}$ and disjoint atoms $A_{1} \ldots, A_{n}$ of $\mathcal{A}_{1}$ with $A=\bigcup_{j=1}^{n} A_{j}$.

Proof. Let $E\left(\nu, \mathcal{A}_{1}\right)=E_{\sigma}\left(\nu, \mathcal{A}_{1}\right)$. We assume that there exists an atom $A$ of $\mathcal{A}$ with $\nu(A)>0$ and disjoint $A_{n} \in \mathcal{A}_{1} \backslash\{\emptyset\}$ with $A=\bigcup_{n \in \mathbb{N}} A_{n}$. We apply Theorem 1 (vi) and obtain some finite $N \subset \mathbb{N}$ with $\nu_{*}\left(\bigcup_{n \in N} A_{n}\right)=\nu(A)$. Therefore there exists some $\tilde{A} \in \mathcal{A}_{1}$ with $\tilde{A} \subset \bigcup_{n \in N} A_{n}$ and $\nu(\tilde{A})>0$. This gives $\emptyset \neq \tilde{A} \subset A$ which is a contradiction.

To prove the converse, we show that condition $(v i)$ of Theorem 1 is fulfilled. Let $A \in \mathcal{A}$ be a $\nu$-atom and $\left(A_{n}\right)_{n \in \mathbb{N}} \subset \mathcal{A}_{1}$ be disjoint with $A=\bigcup_{n \in \mathbb{N}} A_{n}$. We choose an atom $\tilde{A}$ of $\mathcal{A}$ with $\tilde{A} \subset A$ and $\nu(\tilde{A})=\nu(A)$. As $\tilde{A}$ can be written as a finite union of atoms of $\mathcal{A}_{1}$, in $\tilde{A}=\bigcup_{n \in \mathbb{N}}\left(\tilde{A} \cap A_{n}\right)$ only finitely many sets on the right side are nonempty. Thus with $N:=\left\{n \in \mathbb{N}: \tilde{A} \cap A_{n} \neq \emptyset\right\}$ we obtain $\nu_{*}\left(\bigcup_{n \in N} A_{n}\right) \geq \nu(\tilde{A})=$ $\nu(A)$ which was to be shown.

Remark 2 The following example shows that the corollary is not true for arbitrary $\mathcal{A}$, even if $\mathcal{A}$ is atomic: Let $\mathcal{A}$ be the $\sigma$-algebra of all countable subsets of $[0,1]$ and their complements, $\nu$ the restriction of the Lebesgue-measure to $\mathcal{A}$ and $\mathcal{A}_{1}$ the Borel$\sigma$-algebra. Then $\nu(A)=0$ holds for every atom $A$ of $\mathcal{A}$, but ex $E_{\sigma}\left(\nu, \mathcal{A}_{1}\right)=\emptyset$ (see [14], Example 1). With ex $E_{\sigma}\left(\nu, \mathcal{A}_{1}\right)=\operatorname{ex} E\left(\nu, \mathcal{A}_{1}\right) \cap E_{\sigma}\left(\nu, \mathcal{A}_{1}\right)$ and $\operatorname{ex} E\left(\nu, \mathcal{A}_{1}\right) \neq \emptyset$ we get $E\left(\nu, \mathcal{A}_{1}\right) \neq E_{\sigma}\left(\nu, \mathcal{A}_{1}\right)$. For an example with $E_{\sigma}\left(\nu, \mathcal{A}_{1}\right)=\emptyset$ see $[10]$, p. 444 445. 


\section{Extensions of two-valued measures}

The preceeding theorem can be applied to two-valued measures, as in that case every set in $\mathcal{A} \backslash \mathcal{N}(\nu)$ is a $\nu$-atom.

Theorem 2 Let $\mathcal{A} \subset \mathcal{A}_{1}$ be $\sigma$-algebras and $\nu \in c a_{+}(\mathcal{A})$ with $R(\nu)=\{0,1\}$. Then the following conditions are equivalent:

(i) $E\left(\nu, \mathcal{A}_{1}\right)=E_{\sigma}\left(\nu, \mathcal{A}_{1}\right)$.

(ii) $\left\{\mu \in E\left(\nu, \mathcal{A}_{1}\right): R(\mu)=\{0,1\}\right\} \subset E_{\sigma}\left(\nu, \mathcal{A}_{1}\right)$.

(iii) For each decreasing sequence $A_{1} \supset A_{2} \supset \ldots$ in $\mathcal{A}_{1}$ with $\bigcap_{n \in \mathbb{N}} A_{n} \in \mathcal{N}(\nu)$ there exists an $n \in \mathbb{N}$ with $A_{n} \in \mathcal{N}(\nu)$.

(iv) There exists a finite partition $\mathcal{K}$ of $\Omega$ with $\mathcal{A}_{1}=\left(\left(\mathcal{A}_{1} \cap \mathcal{N}(\nu)\right) \cup \mathcal{K}\right)_{\beta}$.

(v) $\operatorname{ex} E\left(\nu, \mathcal{A}_{1}\right)$ is a finite set.

(vi) There exists some $n \in \mathbb{N}$ such that for every $\mu \in E\left(\nu, \mathcal{A}_{1}\right)$ the set $R(\mu)$ contains at most $n$ elements.

(vii) For every $\mu \in E\left(\nu, \mathcal{A}_{1}\right) \cap$ at $\left(\mathcal{A}_{1}\right)$ the set $R(\mu)$ is finite.

Proof. The conditions $(i)$ - (iii) are reformulations of the corresponding conditions in Theorem 1, for (ii) we use Plachky's extremality criterion ([13], Theorem 1).

$($ iii $) \Rightarrow($ iv $)$ : Similar to the proof of Lemma 2, we can define an equivalence relation on the $\left(\mathcal{N}(\nu) \cap \mathcal{A}_{1}\right)$-atoms by $A \sim B: \Leftrightarrow A \triangle B \in \mathcal{N}(\nu)$. Let $\mathcal{K}^{\prime}$ be a system of representatives. If $\mathcal{K}^{\prime}$ contained an infinite sequence $\left(A_{n}\right)_{n \in \mathbb{N}}$, then $B_{n}:=\bigcup_{j=n}^{\infty} A_{j}$ would be a sequence with $\bigcap_{n \in \mathbb{N}} B_{n} \in \mathcal{N}(\nu)$, and (iii) implies $B_{n} \in \mathcal{N}(\nu)$ and therefore $A_{n} \in \mathcal{N}(\nu)$ for some $n \in \mathbb{N}$, which is a contradiction. This shows that $\mathcal{K}^{\prime}$ is finite. We define the partition $\mathcal{K}$ of $\Omega$ as in the proof of Lemma 2. Now let $A \in \mathcal{A}_{1}$ be arbitrary and $K \in \mathcal{K}$. If $A \cap K \notin \mathcal{N}(\nu)$ we have $K \backslash A \in \mathcal{N}(\nu)$ and therefore $A \cap K=K \backslash(K \backslash A) \in\left(\left(\mathcal{N}(\nu) \cap \mathcal{A}_{1}\right) \cup \mathcal{K}\right)_{\beta}$. Now $A=\bigcup_{K \in \mathcal{K}}(A \cap K)$ shows $\mathcal{A}_{1}=\left(\left(\mathcal{N}(\nu) \cap \mathcal{A}_{1}\right) \cup \mathcal{K}\right)_{\beta}$.

$(i v) \Rightarrow(v)$ : If $\mathcal{K}$ is a partition like in $(i v)$ with $n$ elements, ex $E\left(\nu, \mathcal{A}_{1}\right)$ has at most $n$ elements.

$(v) \Rightarrow(v i)$ : Let $\operatorname{ex} E\left(\nu, \mathcal{A}_{1}\right)=\left\{\mu_{1}, \ldots, \mu_{n}\right\}$. Using the integral representation (1), we see that every $\mu \in E\left(\nu, \mathcal{A}_{1}\right)$ can be written in the form $\mu=\sum_{j=1}^{n} \alpha_{j} \mu_{j}$ with $\alpha_{j} \geq 0, \sum \alpha_{j}=1$. Therefore $R(\mu)$ has at most $2^{n}$ elements (cf. [1], section 11.1).

$(v i) \Rightarrow(v i i)$ is trivial.

(vii) $\Rightarrow\left(\right.$ iii): Suppose $($ iii $)$ is false. Then there is a decreasing sequence $\left(A_{n}\right)_{n \in \mathbb{N}} \in$ $\mathcal{A}_{1} \backslash \mathcal{N}(\nu)$ with $\bigcap_{n \in \mathbb{N}} A_{n} \in \mathcal{N}(\nu)$. We define $\mathcal{K}^{\prime}:=\left\{A_{n} \backslash A_{n+1}: A_{n} \backslash A_{n+1} \notin \mathcal{N}(\nu)\right\} . \mathcal{K}^{\prime}$ is infinite. (If this set were finite, we would have some $m \in \mathbb{N}$ with $A_{n} \backslash A_{n+1} \in \mathcal{N}(\nu)$ for all $n \geq m$ and $A_{m}=\bigcup_{n \geq m}\left(A_{n} \backslash A_{n+1}\right) \cup\left(\bigcap_{n \in \mathbb{N}} A_{n}\right) \in \mathcal{N}(\nu)$.) Fix $\tilde{K} \in \mathcal{K}^{\prime}$ and define

$$
\mathcal{K}:=\left\{\left(\Omega \backslash \bigcup \mathcal{K}^{\prime}\right) \cup \tilde{K}\right\} \cup\left\{K \in \mathcal{K}^{\prime}: K \neq \tilde{K}\right\}
$$


As $\mathcal{K}^{\prime}$ is a subset of $\mathcal{A}_{1} \backslash \mathcal{N}(\nu)$, the same is true for $\mathcal{K}$. Moreover, $\mathcal{K}=\left\{K_{1}, K_{2}, \ldots\right\}$ is a countably infinite partition of $\Omega$. By $\mu_{0}\left(\bigcup_{n \in \mathbb{N}}\left(A_{n} \cap K_{n}\right)\right):=\sum_{n \in \mathbb{N}}\left(\frac{1}{2}\right)^{n} \nu\left(A_{n}\right)$ we define an atomic measure extension of $\nu$ to $(\mathcal{A} \cup \mathcal{K})_{\beta}=\left\{\bigcup_{n \in \mathbb{N}}\left(A_{n} \cap K_{n}\right): A_{n} \in \mathcal{A}\right\}$, cf. [2], Satz 2A. Choose $\mu \in \operatorname{ex} E\left(\mu_{0}, \mathcal{A}_{1}\right)$. Using (2) with $\mu_{0}$ instead of $\nu$ we see $\mu \in E\left(\nu, \mathcal{A}_{1}\right) \cap$ at $\left(\mathcal{A}_{1}\right)$. But $R(\mu)=R\left(\mu_{0}\right)$ is infinite which contradicts (vii).

Remark 3 For arbitrary $\nu \in c a_{+}(\mathcal{A})$, condition $(i v)$ of the preceeding theorem implies condition $(i)$. This follows from [7], Remark 2, since the completion of $\nu$ is $\sigma$-additive.

Acknowledgement. The authors express their gratitude to Prof. Z. Lipecki who drew their attention to the problem and gave valuable hints and comments.

\section{References}

[1] K. P. S. Bhaskara Rao, M. Bhaskara Rao: Theory of charges. A study of finitely additive measures. Academic Press London 1983.

[2] D. Bierlein: Über die Fortsetzung von Wahrscheinlichkeitsfeldern. Z. Wahrsch. Verw. Gebiete 1 (1962), 28 - 46.

[3] D. Bierlein, W. J. A. Stich: On the extremality of measure extensions. Manuscripta Math. 63 (1989), 89 - 97.

[4] D. Bogner: Simultane $\sigma$-Additivität in Klassen von Inhaltsfortsetzungen eines Maßes. Diploma thesis, Universität Regensburg, 1991.

[5] N. Dunford, J. T. Schwartz: Linear Operators, Part I: General Theory. Interscience Publishers New York 1957.

[6] J. Kindler: The sigma-core of convex games and the problem of measure extensions. Manuscripta Math. 66 (1989), 97 - 108.

[7] J. Lembcke: On a measure extension theorem of Bierlein. Lecture Notes in Math. 794, $45-48$.

[8] Z. Lipecki, private communication.

[9] Z. Lipecki: Decomposition theorems for Boolean rings, with applications to semigroupvalued measures. Comment. Math. Prace Mat. 20 (1978), 397 - 403.

[10] Z. Lipecki: A generalization of an extension theorem of Bierlein to group-valued measures. Bull. Acad. Polon. Sci. Sér. Sci. Math. 28 (1980), 441 - 445.

[11] Z. Lipecki: On extreme extensions of quasi-measures. Arch. Math. 58 (1992), 288 293.

[12] R. R. Phelps: Lectures on Choquet's theorem. D. von Nostrand Company, Princeton 1966.

[13] D. Plachky: Extremal and monogenic additive set functions. Proc. Amer. Math. Soc. 54 (1976), 193 - 196.

[14] D. Plachky: Darboux property of measures and contents. Math. Slovaca 30 (1980), $243-246$. 